\title{
On the Morphological Processing of Objects with Varying Local Contrast*
}

\author{
Pierre Soille \\ EC Joint Research Centre \\ Institute for Environment and Sustainability \\ Land Management Unit \\ TP 262, I-21020 Ispra, Italy \\ Pierre.Soille@jrc.it
}

\begin{abstract}
Most morphological operators appear by pairs such as erosion/dilation, opening/closing, and thinning/thickening. These are pairs of dual operators with respect to set complementation. The output of a (dual) morphological operator applied to an object depends on whether it is a bright object over a dark background or a dark object over a bright background. When dealing with complex images such as earth observation data, there is no clear distinction between the background and the foreground because the image consists of a partition of the space into image objects of arbitrary intensity values. In this paper, we present an overview of existing approaches for tackling this problem and propose new techniques based on area filters applied first to the image extrema and then to all flat regions.
\end{abstract}

Keywords: Mathematical morphology, self-duality, self-complementarity, partition, region growing, flat regions, compression, satellite images.

\section{Introduction}

Most morphological operators appear by pairs such as erosion/dilation $(\varepsilon, \delta)$, opening/closing $(\gamma, \phi)$, and thinning/thickening (THIN, THICK). These are pairs of dual operators with respect to set complementation. Rather than pairs of dual operators, we could have referred to the more general concept of pairs of adjunct operators. However, because we restrict our attention to morphological operators applied to grey scale images, the notion of duality suits our needs. In mathematical terms, two image transformations $\Psi$ and $\Phi$ are dual with respect to complementation if applying $\Psi$ to an image is equivalent to applying $\Phi$ to the complement of the image and taking the complement of the result:

$\Psi$ and $\Phi$ are dual with respect to complementation $\complement \Leftrightarrow \Psi=\complement \Phi \complement$.

For example, dilating an image is equivalent to eroding the complement of this image and then complement the output eroded image: $\delta=\complement \varepsilon \complement$. Although the

\footnotetext{
* This work was supported by the EC-JRC ESDI Project.
} 
duality principle is fundamental to many morphological operators, its implications are often overlooked. Indeed, the output of a (dual) morphological operator applied to an object depends on whether it is a bright object over a dark background or a dark object over a bright background. This is not a major issue for applications where the local contrast of a given object type does not vary over the image. It happens for instance with images of cells whose nuclei usually appear darker than their cytoplasm, man-made objects controlled by machine vision systems, etc. However, when dealing with more complex images such as earth observation data, there is no clear distinction between the background and the foreground because the image consists of a partition of the space into arbitrary image objects. As a consequence, an object such as a field may appear darker or brighter than the surrounding fields depending on the reflectance of its neighbouring fields [9]. In addition, satellite images are often multichannel images. It follows that the independent processing of each channel by a dual operator may lead to inconsistent results because an object may appear darker than its neighbourhood in a channel and vice versa in another. A further relevant application is the filtering of speckle noise. Indeed, owing to their symmetric nature, constructive and destructive interferences should be processed identically.

A solution to mitigate the non-symmetric behaviour of dual morphological operator is to apply, in a sequence, a filter and then its dual. For instance, one may apply an opening followed by a closing (or vice versa), starting with the smallest possible size and proceed until the maximum filter size is reached. This idea is at the basis of alternating sequential filters. However, although alternating sequential filters process bright and dark structures much more equally than the mere utilisation of a unique opening or closing, their output usually depends on whether one starts the sequence with an opening or a closing.

In situations where a strict symmetric processing of objects either brighter or darker than their neighbourhood is required, self-dual operators should be considered. In mathematical terms, an image-to-image transformation $\Psi$ is selfdual with respect to the complementation operator $\complement$ if its dual transformation with respect to complementation is itself:

$$
\Psi \text { is self-dual with respect to complementation } \complement \Leftrightarrow \Psi=\complement \Psi \complement \text {. }
$$

The complement of an image $f$, denoted by $f^{c}$, is defined for each pixel $\mathbf{x}$ as the maximum value of the data type used for storing the image $t_{\max }$ minus the value of the image $f$ at position $\mathbf{x}$, i.e., $f^{c}(\mathbf{x})=t_{\max }-f(\mathbf{x})=[\complement(f)](\mathbf{x})$. For example, normalised shift-invariant operators are also self-dual operators. The median filter is an example of non-linear self-dual filter.

Motivated by applications dealing with objects whose local contrast may vary across the image, we propose to analyse the behaviour of morphological operators for such variations. The paper is organised as follows. A brief review of morphological self-dual operators including new developments related to the link between switch operators and thinning/thickening operations is given in Sec. 2. We show in Sec. 3 that self-duality is not sufficient for some applications and discuss possible techniques addressing this problem. 


\section{Morphological Self-dual Operators}

Self-dual operators defined in terms of switch operators are recalled in Sec. 2.1. We then show in Sec. 2.2 that there exist equivalent representations in terms of thinning and thickening. We finally describe in Sec. 2.3 self-dual alternating sequential filters based on area opening and closing.

\subsection{Switch-Based Self-dual Operators [4]}

A thorough paper about the notion of self-duality in mathematical morphology is due to Heijmans [4]. This paper was motivated by the search for a representation of self-dual morphological increasing and idempotent operators. Because increasing self-dual activity-extensive ${ }^{1}$ operators converge when iterated, the problem comes down to find a representation of the latter operators. This is achieved step by step, starting from an arbitrary self-dual operator and progressively constraining it with the desired properties of a morphological filter (increasingness, translation invariance, and idempotency). These developments are summarised in this section for increasing and translation invariant self-dual operators (i.e., without taking into account the idempotency property).

Consider an arbitrary self-dual operator $\Xi$ applied to an arbitrary set $X$. We denote by $\sigma(X)$ the points of $X$ which are suppressed by $\Xi$, i.e., $\sigma(X)=$ $X \backslash \Xi(X)=X \cap(\Xi(X))^{c}=X \cap \Xi\left(X^{c}\right)$. It follows that $\Xi$ can be written in terms of $\sigma$ as follows, id denoting the identity operator:

$$
\Xi=(\mathrm{id} \wedge \complement \sigma) \vee \sigma \complement \text {. }
$$

An anti-extensive operator $\sigma$ leading to a self-dual increasing operator is called a switch operator and satisfies the following two conditions [4, p. 22]:

$$
\begin{array}{r}
X \subseteq Y \Rightarrow X \cap \sigma(Y) \subseteq \sigma(X), \\
\sigma(X \cup\{\mathbf{h}\}) \cap \sigma\left(X^{c} \cup\{\mathbf{h}\}\right)=\emptyset, \quad \mathbf{h} \in \mathbb{Z}^{2}, X \in \mathbb{Z}^{2} .
\end{array}
$$

Recall that any increasing operator extends directly to grey tone images using the threshold superposition principle which states that the output of an increasing operator applied to a grey tone image equals the sum its binary cross-sections processed by this operator. The adjective 'switch' indicates that the operator $\sigma$ yields all points which switch value from 1 to 0 (points in $\sigma(X)$ ) or from 0 to 1 (points in $\sigma\left(X^{c}\right)$ ) by application of the corresponding self-dual increasing operator $\Xi: \Xi(X)=(X \backslash \sigma(X)) \cup \sigma\left(X^{c}\right)$. By considering the additional translationinvariance property, the basis representation of a translation invariant increasing operator [3] leads to a representation of switch operators as well as their corresponding translation invariant increasing self-dual operators. More precisely, every translation invariant switch $\sigma_{\mathcal{A}}$ can be written as follows [4, p. 25]:

${ }^{1}$ An operator $\Psi_{1}$ is less active than an operator $\Psi_{2}$ if for all pixels of any given input image, the output value of $\Psi_{1}$ at any given pixel lies in the interval defined by the input value and the output of $\Psi_{2}$ at this pixel. An operator $\Psi$ is called activityextensive if $\Psi$ iterated $n$ times is less active that $\Psi$ iterated $n+1$ times. 


$$
\sigma_{\mathcal{A}}=\mathrm{id} \wedge \bigvee_{A \in \mathcal{A}} \varepsilon_{A} \complement
$$

where $\mathcal{A}$ is a collection of structuring elements such that the origin o belongs to none of them (i.e., $\mathbf{o} \notin A$ for all $A \in \mathcal{A}$ ) and the intersection of any two structuring elements is non-empty (i.e., $A \cap B \neq \emptyset$ for all $A, B \in \mathcal{A}$ ). The representation of the corresponding self-dual operator $\Xi_{\mathcal{A}}$ is obtained by substituting $\sigma$ with $\sigma_{\mathcal{A}}$ in Eq. 3 and simplifying the right term:

$$
\Xi_{\mathcal{A}}=\left(\mathrm{id} \wedge \bigwedge_{A \in \mathcal{A}} \delta_{A}\right) \vee \bigvee_{A \in \mathcal{A}} \varepsilon_{A}
$$

\subsection{Link between Switch and Thinning/Thickening Representations}

We now show that Eq. 7 can be expressed in terms of thinnings and thickenings as exemplified in [8, Fig. 5.12, p. 157] for the special case of a self-dual filter removing isolated pixels of grey scale images. Indeed, the left part of Eq. 7 corresponds to an intersection of thinnings performed in parallel with a series of composite structuring elements $\mathbf{B i}=\left(B i_{1}, B i_{2}\right)$ such that $B i_{1}$ is restricted to the origin $\mathbf{o}$ and the sets $B i_{2}$ form a one-to-one correspondence with the sets $A$ of $\mathcal{A}$ :

$$
\begin{aligned}
\bigcap_{\mathbf{B i}} \operatorname{THIN}_{\mathbf{B i}}(X) & =\bigcap_{\mathbf{B i}}\left[X \backslash \operatorname{HMT}_{\mathbf{B i}}(X)\right] \\
& =\bigcap_{\mathbf{B i}}\left[X \cap\left[\operatorname{HMT}_{\mathbf{B i}}(X)\right]^{c}\right] \\
& =\bigcap_{\mathbf{B i}}\left[X \cap\left[\varepsilon_{B i_{1}}(X) \cap \varepsilon_{B i_{2}}\left(X^{c}\right)\right]^{c}\right] \\
& =\bigcap_{\mathbf{B i}}\left[X \cap\left[\delta_{B i_{1}}\left(X^{c}\right) \cup \delta_{B i_{2}}(X)\right]\right] \\
& =\bigcap_{B i_{2}}\left[X \cap \delta_{B i_{2}}(X)\right] \\
& =X \cap \bigcap_{B i_{2}} \delta_{B i_{2}}(X) \\
& =X \cap \bigcap_{A \in \mathcal{A}} \delta_{A}(X),
\end{aligned}
$$

where HMT denotes the hit-or-miss transformation. This result can also be obtained starting from the observation $\left[4\right.$, p. 26] that $\sigma_{\mathcal{A}}$ corresponds to a union of hit-or-miss transformations: $\sigma_{\mathcal{A}}=\bigcup_{A \in \mathcal{A}} \mathrm{HMT}_{\mathbf{B}=(\{\mathbf{o}\}, A)}$. Indeed, it follows that: 


$$
\begin{aligned}
\mathrm{id} \wedge \complement \sigma_{\mathcal{A}} & =\mathrm{id} \wedge \complement \bigcup_{A \in \mathcal{A}} \operatorname{HMT}_{(\{\mathbf{o}\}, A)} \\
& =\mathrm{id} \wedge \bigcap_{A \in \mathcal{A}} \operatorname{CHMT}_{(\{\mathbf{o}\}, A)} \\
& =\bigcap_{A \in \mathcal{A}}\left[\mathrm{id} \wedge \operatorname{CHMT}_{(\{\mathbf{o}\}, A)}\right] \\
& =\bigcap_{A \in \mathcal{A}} \operatorname{THIN}_{(\{\mathbf{o}\}, A) .}
\end{aligned}
$$

Note that in this paper, the constrained version of the hit-or-miss and thinning operators [7] must always be considered when processing grey level images. Remember that the right term of Eq. 7 (as well as the corresponding term in Eq. 3) corresponds to pixels switching from 1 to 0 when $\sigma$ is applied to $X^{c}$. That is, some pixels of the background of $X$ are unioned to the intersection of thinnings. Now, because $\Xi$ is self-dual, an equivalent formulation of Eq. 3 is as follows:

$$
\Xi=(\mathrm{id} \vee \sigma \complement) \wedge \complement \sigma,
$$

and, accordingly,

$$
\Xi_{\mathcal{A}}=\left(\mathrm{id} \vee \bigvee_{A \in \mathcal{A}} \varepsilon_{A}\right) \wedge \bigwedge_{A \in \mathcal{A}} \delta_{A}
$$

Observing that $\operatorname{HMT}_{\mathbf{B}}(X)$ equals $\operatorname{HMT}_{\mathbf{B}^{c}}\left(X^{c}\right)$ where $\mathbf{B}=\left(B_{1}, B_{2}\right)$ and $\mathbf{B}^{c}=$ $\left(B_{2}, B_{1}\right)$, the left term of Eq. 8 can be decomposed as follows:

$$
\begin{aligned}
\operatorname{id} \vee \sigma_{\mathcal{A}} \complement & =\operatorname{id} \vee \bigcup_{A \in \mathcal{A}} \operatorname{HMT}_{(\{\mathbf{o}\}, A)} \complement \\
& =\operatorname{id} \vee \bigcup_{A \in \mathcal{A}} \operatorname{HMT}_{(A,\{\mathbf{o}\})} \\
& =\bigcup_{A \in \mathcal{A}}\left[\operatorname{id} \vee \operatorname{HMT}_{(A,\{\mathbf{o}\})}\right] \\
& =\bigcup_{A \in \mathcal{A}} \operatorname{THICK}_{(A,\{\mathbf{o}\})} .
\end{aligned}
$$

We now show that when this union of thickening is altering (increasing) the intensity value of a given pixel, the subsequent intersection with $\bigwedge_{A \in \mathcal{A}} \delta_{A}$ appearing in Eq. 9 will never further alter this value (equivalent developments apply to the thinning representation corresponding to the left term of Eq. 7).

Proof. The union of thickening at a given position $\mathbf{x}$ of an image $f$ modifies the value of the input image at this position if and only if $\left[\bigvee_{A \in \mathcal{A}} \varepsilon_{A}(f)\right](\mathbf{x})>f(\mathbf{x})$. This implies that there exists a $A^{\star} \in \mathcal{A}$ such that $f(\mathbf{x}+\mathbf{a})>f(\mathbf{x})$ for all $\mathbf{a} \in A^{\star}$ and $\left[\bigvee_{A \in \mathcal{A}} \varepsilon_{A}(f)\right](\mathbf{x})=\wedge_{\mathbf{a} \in A^{\star}} f(\mathbf{x}+\mathbf{a})$. Because $A \cap B \neq \emptyset$ for all $A, B \in \mathcal{A}$, for all $B \in \mathcal{A}$ there exists a $\mathbf{a} \in A^{\star}$ such that $\mathbf{a} \in B$. This implies that $\left[\bigwedge_{A \in \mathcal{A}} \delta_{A}(f)\right](\mathbf{x}) \geq\left[\bigvee_{A \in \mathcal{A}} \varepsilon_{A}(f)\right](\mathbf{x})$. Indeed, for all $B \in \mathcal{A}, \mathbf{a} \in B \cap A^{\star}$, the following inequality holds: $\vee_{\mathbf{b} \in B} f(\mathbf{x}+\mathbf{b}) \geq f(\mathbf{x}+\mathbf{a})$. 
Consequently $\Xi_{\mathcal{A}}$ reduces to the anti-centre based on the intersection of thinnings and union of thickenings.

\subsection{Area Based Self-dual Filters and Self-dual Reconstruction}

Specific compositions of 8-connected area opening and closing lead to self-dual morphological filters. More precisely, the composition of an 8-connected area opening $\gamma_{\lambda}^{8}$ with the dual area closing $\phi_{\lambda}^{8}$ and area parameter $\lambda$ equal to 2 is a self-dual morphological filter. This filter can also be expressed in terms of a switch operator with the 8-neighbour ring as structuring element (SE) and the origin at its centre. Its formulation in terms of thinnings and thickenings is illustrated in [8, Fig. 5.12, p. 157]. More generally, it can be shown that the open-close filters, based on 8-connected area filters, are self-dual up to an area of 8 pixels and are identical to the corresponding close-open filters:

$$
\phi_{\lambda}^{8} \gamma_{\lambda}^{8}=\gamma_{\lambda}^{8} \phi_{\lambda}^{8}, \forall \lambda \in\{2,3, \ldots, 8\}
$$

For larger sizes, alternating sequential filters (ASFs) based on 8-connected area closings and openings lead to self-dual filters. From a computational point of view, it is worth mentioning that, for an 8-connected area ASF of size $n$ larger than 20, only sizes 8,16,20 need to be considered and then every even size smaller than $n$, and finally the size $n$. For example, it can be shown that the following equalities holds:

$$
\begin{aligned}
\gamma_{25}^{8} \phi_{25}^{8} \cdots \gamma_{3}^{8} \phi_{3} \gamma_{2}^{8} \phi_{2} & =\phi_{25}^{8} \gamma_{25}^{8} \cdots \phi_{3}^{8} \gamma_{3} \phi_{2}^{8} \gamma_{2}= \\
\gamma_{25}^{8} \phi_{25}^{8} \gamma_{24}^{8} \phi_{24}^{8} \gamma_{22}^{8} \phi_{22}^{8} \gamma_{20}^{8} \phi_{20}^{8} \gamma_{16}^{8} \phi_{16}^{8} \gamma_{8}^{8} \phi_{8}^{8} & =\phi_{25}^{8} \gamma_{25}^{8} \phi_{24}^{8} \gamma_{24}^{8} \phi_{22}^{8} \gamma_{22}^{8} \phi_{20}^{8} \gamma_{20}^{8} \phi_{16}^{8} \gamma_{16}^{8} \phi_{8}^{8} \gamma_{8}^{8} .
\end{aligned}
$$

The latter filter is illustrated in Fig. 1 on a satellite image. In this experiment, the three channels of the input multichannel image have been processed independently.

\section{Beyond Self-duality}

In some applications, self-duality is only partially addressing the problem of treating equally objects with varying local contrast. Indeed, self-dual filters such as the alternating sequential filters described in Sec. 2.2 are still assuming that the targeted image objects are either brighter or darker than the surrounding objects (i.e., we assume that the targeted image structures are marked by image extrema). While this is a valid assumption for processing objects such as speckle patterns, it does not apply to image structures showing more than two phases such as satellite images with various crop fields or microscopy images of rock samples with various minerals. In these situations, objects correspond to regions of homogeneous grey scale rather than simply maxima and minima. As a consequence, a self-dual filter such the alternating sequential filters described in Sec. 2.2 is not robust in the sense that it will only alter those regions that 


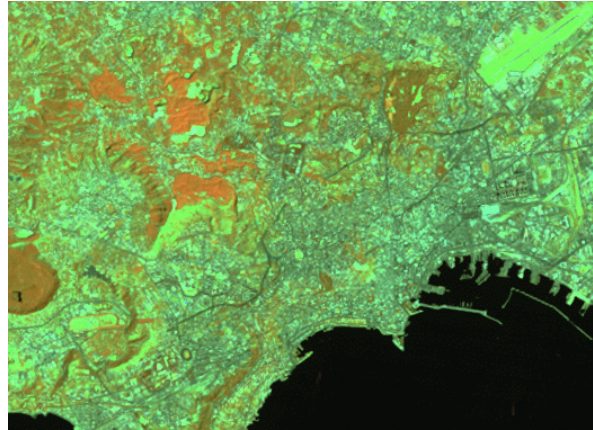

(a) $521 \times 381$ Landsat image of Naples: false RGB colour composite using bands 4,5 , and 7 .

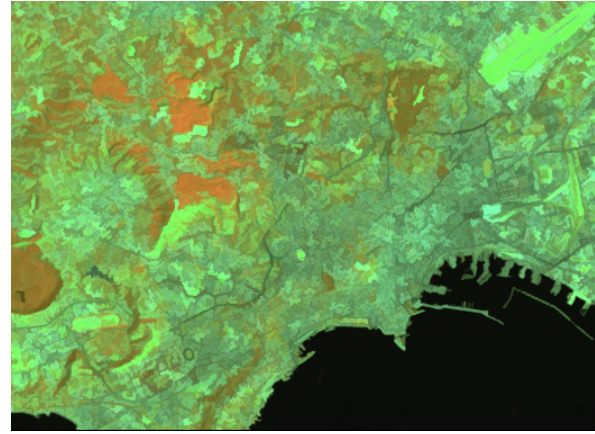

(b) 8-connected self-dual area ASF up to an area 25 pixels.

Fig. 1. Self-dual image simplification removing all dark and bright connected components of pixels smaller than a given threshold. This is achieved by performing the alternating sequential filter based on 8-connected area openings and closings (as per Eq. 11).

are completely surrounded by either darker or brighter regions. That is, intermediate regions occurring as plateaus may remain unaffected. We present in this section two approaches tackling this problem. The first (Sec. 3.1) consists in applying self-complementary operators while the second (Sec. 3.2) is based on the processing of the image flat zones.

\subsection{Self-complementary Based Operators}

Self-complementary operators may be considered for processing image objects independently of their local contrast. Indeed, a self-complementary operator $\Phi$ outputs the same result if applied to an image or its complement [8, p. 55]:

$$
\Phi \text { is self-complementary with respect to } \complement \Leftrightarrow \Phi=\Phi \complement \text {. }
$$

For example, both the morphological gradient (arithmetic difference between the dilation and erosion of an image) and the norm of a gradient computed using derivative convolution kernels are self-complementary operators. Consequently, morphological segmentation techniques such as those based on the watershed transformation of self-complementary gradients guarantee that the resulting segmentation is independent of the local contrast of the searched objects in the input image (assuming the marker set are extracted accordingly). Moreover, contrary to self-dual filters, plateaus are treated identically to image extrema by the gradient operator. Indeed, a plateau region such as a field adjacent to both darker and brighter fields may remain unaffected by a self-dual filter although this latter filter may remove a similar field appearing as an image extremum, i.e., a field surrounded by either darker or brighter fields.

However, a drawback of a gradient based processing is that the searched regions of the image must be thick enough to display a core with low gradient 
values. This problem is caused by the the limited resolution of the gradient. It has motivated Crespo et al. [2] to propose an alternative approach based on the merging of flat zones. Similarly, Pesaresi and Benediktsson [5] proposed the notion of morphological profiles to avoid this resolution problem when segmenting satellite images.

\subsection{Sequential Area Filtering of the Image Flat Zones}

Owing to their very nature, area opening and closing act only on the image extrema. It follows that transition regions or intermediate plateaus may be preserved by these filters even if their extent is smaller than the selected area parameter. This issue is illustrated in Fig. 2 by displaying the partition of the satellite image filtered by an area opening and closing corresponding to Fig. 1b.

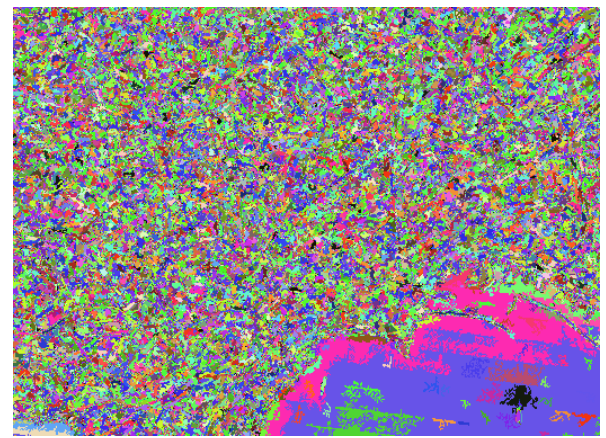

Fig. 2. Flat zones of the image shown in Fig. 1b (alternating self-dual area opening and closing up to an area of 25 pixels). Although this filter ensures that all extrema of the filtered image are larger or equal to the size of the filter, flat zones belonging to nonextrema regions can be of arbitrary size in the filtered image. Flat zones corresponding to the first channel of the processed multichannel satellite image are displayed.

We propose to simplify the image by removing all flat zones whose area is below a given threshold value as follows:

- First extract all flat zones whose area is greater than or equal to a given threshold value. This is achieved by labelling the flat zones of the initial image according to the fast breadth first stack based algorithm described in [8, p. 38]. Those labelled regions whose area are equal to or exceed the threshold value are then selected.

- Define an ordered procedure to grow the selected flat zones while preserving their initial grey level values so as to obtain a new partition of the image definition domain into flat zones. This is achieved by adapting the seeded region growing algorithm described in [1]. 
Similarly to alternating sequential filters, a better preservation of the relevant image structures is obtained by iterating the proposed area based filtering for increasing values of the area threshold level until the desired value is reached. For example, Fig. 3a shows the output of the proposed filtering by iterating it up to an area threshold of 25 pixels and using 8-connectivity. Contrary to the

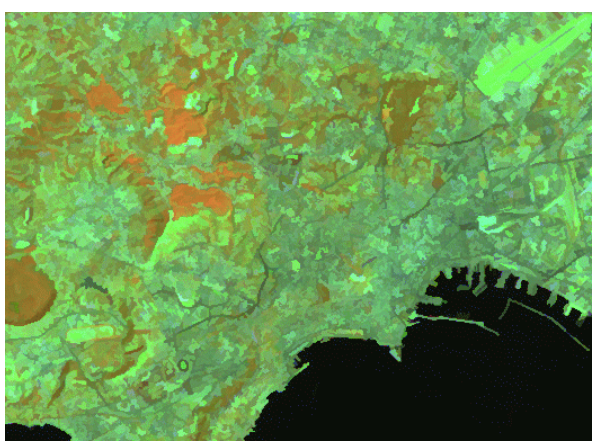

(a) 8-connected area sequential filter applied to Fig. 1a for an area of up to 25 pixels.

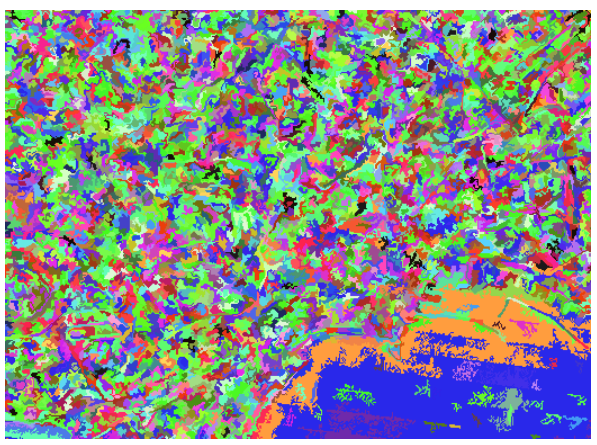

(b) Corresponding image partition (for the first channel).

Fig. 3. Sequential area filtering of the flat zones of the multichannel satellite image displayed in Fig. 1a. Compare the image displayed in (a) with the output of the alternating sequential area opening/closing filter displayed in Fig. 1b as well as the corresponding flat zones partitions of the first channel.

partition produced by the alternating sequential area opening/closing filter (see Fig. 2), each flat zone of the alternating area filter of the flat zones up to an area of $\lambda$ pixels has at least $\lambda$ pixels (Fig. 3b).

Note that Salembier et al. [6] also propose a filter suppressing all flat regions whose area is less than a given threshold. It is based on the processing of the region adjacency graph of the flat zones using an area merging criterion and setting the grey level of the merged region to the median value of the largest region (or the arithmetic mean of the two merged regions if they have both the same size) while considering an ad hoc merging order. Contrary to our approach, this type of process defines a connected operator. That is, when a flat zone is below the threshold level, it cannot be shared by two different flat zones.

\section{Conclusion and Perspectives}

Beyond background techniques for generating self-dual morphological filters and new links between switch operators and thinning/thickening pairs, we have focused our attention to new filters based on area filters. The first category is based on area opening and closing. However, it assumes that relevant objects are either brighter or darker than their neighbourhood. This model does not apply to complex images such as satellite images with numerous land cover types. 
We have proposed to address this problem by suppressing all flat zones of the image using an area criterion and then grow the remaining flat zones using a modified seeded region growing technique. We are in the process of evaluating this technique for extracting thematic information from pan-European Landsat imagery.

\section{Acknowledgements}

I wish to thank Henk Heijmans for stimulating discussions about switch operators.

\section{References}

[1] R. Adams and L. Bischof. Seeded region growing. IEEE Transactions on Pattern Analysis and Machine intelligence, 16(6):641-647, 1994.

[2] J. Crespo, R. Schafer, J. Serra, C. Gratin, and F. Meyer. The flat zone approach: a general low-level region merging segmentation method. Signal Processing, 62(1): 37-60, 1997.

[3] H. Heijmans. Morphological Image Operators. Advances in Electronics and Electron Physics Series. Academic Press, Boston, 1994.

[4] H. Heijmans. Self-dual morphological operators and filters. Journal of Mathematical Imaging and Vision, 6:15-36, 1996. URL ftp://ftp.cwi.nl/pub/morphology/ report/Heijmans_selfdual.ps.Z.

[5] M. Pesaresi and J. Benediktsson. A new approach for the morphological segmentation of high resolution satellite imagery. IEEE Transactions on Geoscience and Remote Sensing, 39(2):309-320, February 2001.

[6] P. Salembier, L. Garrido, and D. Garcia. Auto-dual connected operators based on iterative merging algorithms. In H. Heijmans and J. Roerdink, editors, Mathematical Morphology and its Applications to Image and Signal Processing, volume 12 of Computational Imaging and Vision, pages 183-190, Dordrecht, 1998. Kluwer Academic Publishers.

[7] P. Soille. Advances in the analysis of topographic features on discrete images. Lecture Notes in Computer Science, 2301:175-186, March 2002. URL http://link. springer.de/link/service/series/0558/bibs/2301/23010175.htm.

[8] P. Soille. Morphological Image Analysis: Principles and Applications. Springer-Verlag, Berlin Heidelberg New York, 2nd edition, 2003. See also http://ams.jrc.it/soille/book2nd.

[9] P. Soille and M. Pesaresi. Advances in mathematical morphology applied to geoscience and remote sensing. IEEE Transactions on Geoscience and Remote Sensing, 40(9):2042-2055, September 2002. 